\title{
Modeling and Preliminary Design Issues of a 3-axis Parallel Machine-Tool
}

\author{
O. Company, F. Pierrot \\ LIRMM UMR 5506 CNRS - UM2 \\ 161 rue Ada \\ 34392 Montpellier Cedex 5, France
}

\author{
F. Launay, C. Fioroni \\ Renault-Automation Comau \\ ZI de Mélou BP 237 \\ 81104 Castres Cedex, France
}

\begin{abstract}
This paper presents preliminary studies dedicated to a 3-axis PKM intended to be used as a Drilling-Taping machine-tool. We first explain our method to select a suitable parallel mechanism and a convenient general arrangement. Then we derive all the necessary models for preliminary design, including position, kinematics, statics, dynamics and simplified stiffness evaluation models. Finally, some results show the effectiveness of those models.
\end{abstract}

\section{Introduction}

An analysis of the operation times of agile machines reveals the following results: $50 \%$ of time making chips, $50 \%$ for auxiliary tasks. An important question for the new machine-tool generation is how to raise the proportion of chip-making time and to go above $50 \%$ to $60 \%$, maybe even more to approach the ultimate goal of $100 \%$ chip-making time?

According recent studies, different routes to this goal can be explored [1], including:

$\checkmark$ to increase the cutting speed by adopting electric spindles capable of $40,000 \mathrm{rpm}$ or more;

$\checkmark$ to eliminate tool changeover altogether, following the idea that the ultra-agile machine specializes in operations such as drilling, facing, taping and boring which, whatever the diameter of the holes, can be carried out with a single tool, or two or three at the maximum;

$\checkmark$ to increase dynamic performances by a factor of three to four with respect to "classical" high-speed machines. This means reaching accelerations of $35 \mathrm{~m} . \mathrm{s}^{-2}$, even $50 \mathrm{~m} \cdot \mathrm{s}^{-2}$. Only parallel architectures would enabled such values to be attained while minimizing masses and keeping a high stiffness.

The aim of this paper is to present some preliminary studies that have been conducted by Renault-Automation and LIRMM-CNRS as part of a larger project consisting in the construction of a ultra-agile drilling-taping parallel machinetool.

\section{Selection of a suitable arrangement}

A drilling-taping machine requires a mechanism with three degrees of freedom (dof) in translation. From the very beginning of this study we decided not to consider over-actuated machines nor machines with passive chains for simplicity reasons, and to focus only on pure fully-parallel machines, that is machines with three independent chains including one actuator per chain. Few parallel mechanisms provided this, namely the Delta structure [2] invented by Clavel, the machine proposed by Tsai [3], the Star [4] or the Prism structure [5] proposed by 
Herve and the machine derived from the Delta proposed by Mitova et al. [6].

In order to obtain high acceleration, it is important to keep the moving objects mass as low as possible: this is a reason to choose the structures based on kinematic chains with the motor fixed on the base; moreover, having motors on the frame is also good to obtain a good thermal behavior. Among the existing solutions, only Delta and Star fulfil this requirement. We have chosen the Delta for two reasons: (i) it has been intensively tested as a robot structure while Star never became a commercial product as a robot, and (ii) its mechanical structure guarantees that the fixed length legs (the "rods") are stressed only in tension-compression.

On the other hand, from technological point of view, it is clear that linear drives can offer the best performance in terms of acceleration. We will then focus our work on Linear DELTA with Linear Drives .

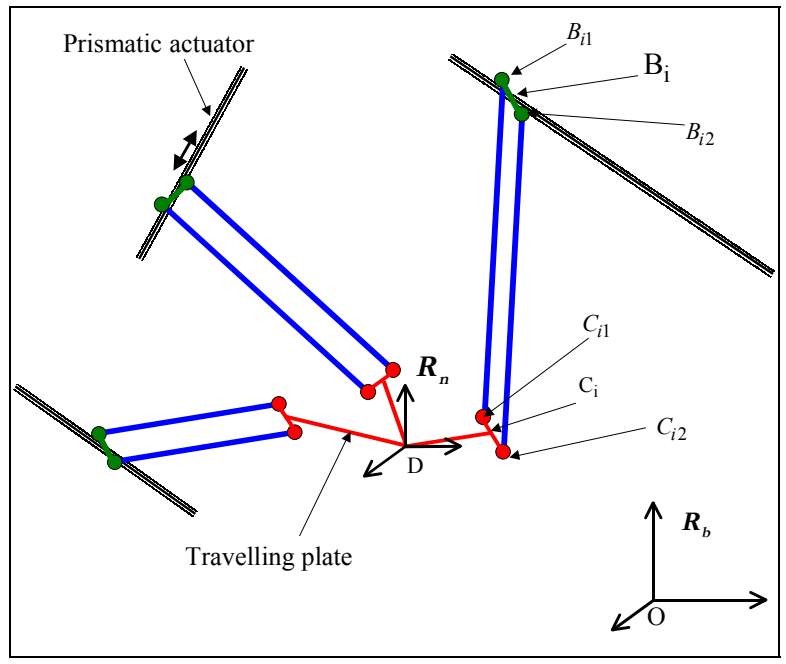

Figure 1 : Linear Delta.

As shown in Figure 1, the mechanism has 3 linear drives, 6 rods grouped by pairs and a travelling plate. As long as some geometric constraints are fulfilled (e.g. the rods in a pair must be parallel to each other) the mechanism will offer 3 translations, whatever the location of prismatic actuated joints.

The first phase of a preliminary design is then to select a convenient arrangement for those actuated joints, before optimizing the rest of the structure.

\subsection{Generic modeling}

Generic models are derived so that they are compatible with all possible geometry of the machine (but not optimal in terms of computational efficiency). We note:

- $\mathrm{P}_{\mathrm{i}}$ a point on prismatic joint axis number $i$;

- $u_{i}$ a unitary vector for prismatic joint number $i$;

- $\mathrm{B}_{\mathrm{i}}$ the point at the middle of ball joints centers (pair number $i$ ) on the actuator side, $\mathrm{B}_{\mathrm{i} 1}$ and $\mathrm{B}_{\mathrm{i} 2}$;

- $\mathrm{C}_{\mathrm{i}}$ the point at the middle of ball joints centers (pair number $i$ ) on the travelling plate side, $\mathrm{C}_{\mathrm{i} 1}$ and $\mathrm{C}_{\mathrm{i} 2}$;

- $\mathrm{D}$ the controlled point onto the travelling plate;

- $\mathrm{R}_{\mathrm{b}}$ a fixed frame of origin $\mathrm{O}$;

- $\mathrm{R}_{\mathrm{n}}$ a moving frame linked to the traveling plate of origin $\mathrm{D}$ (note that $\mathrm{R}_{\mathrm{n}}$ stays parallel to $\mathrm{R}_{\mathrm{b}}$ );

- $l_{i}$ length of the rods of pair number $i$.

\section{Inverse position relationship.}

Let us note $\boldsymbol{q}={ }^{t}\left[\begin{array}{lll}q_{1} & q_{2} & q_{3}\end{array}\right]$ and $\boldsymbol{x}={ }^{t}\left[\begin{array}{lll}x & y & z\end{array}\right]$ the set of joint positions and the vector of Cartesian position, respectively. Then, the position of each actuator is expressed as follows :

$$
\boldsymbol{B}_{\boldsymbol{i}}=\boldsymbol{P}_{\boldsymbol{i}}+q_{i} \boldsymbol{u}_{\boldsymbol{i}}
$$

and vector $\boldsymbol{B}_{i} \boldsymbol{C}_{\boldsymbol{i}}$ can be written as follows:

$$
B_{i} C_{i}=\left(x-P_{i}-C_{i}\right)-\left(q_{i} u_{i}\right)
$$


The position relationship is then expressed as a second order polynomial :

$$
q_{i}^{2}-q_{i}\left[2\left(x-P_{i} C_{i}\right) u_{i}\right]+\left(x-P_{i} C_{i}\right)^{2}-l_{i}^{2}=0
$$

If the travelling plate pose $x$ is reachable, this leads to two real roots:

$$
q_{i}=\left(x-P_{i} C_{i}\right) u_{i} \pm \sqrt{\left[\left(x-P_{i} C_{i}\right) u_{i}\right]^{2}-\left(x-P_{i} C_{i}\right)^{2}+l_{i}^{2}}
$$

\section{Kinematics.}

This section is dedicated to the relationship between actuator velocities $\dot{q}_{i}$ and Cartesian velocities $\dot{x}$. Rather than computing the derivative of the position relationship with respect to time, we recommend to apply the " equiprojectivity " property that states:

$$
\boldsymbol{v}_{\mathrm{B}_{\mathrm{i}}} \cdot \boldsymbol{B}_{i} C_{i}=v_{\mathrm{c}_{\mathrm{i}}} \cdot \boldsymbol{B}_{i} C_{i}
$$

where

$$
\boldsymbol{v}_{\mathrm{B}_{\mathrm{i}}}=\dot{q}_{i} \boldsymbol{u}_{i} \text { and } \boldsymbol{v}_{\mathrm{C}_{\mathrm{i}}}=\boldsymbol{v}_{\mathrm{D}_{\mathrm{i}}}=\dot{\boldsymbol{x}}
$$

Writing this for each chain, and grouping the results in a matrix format leads to:

$$
J_{q} \dot{q}=J_{x} \dot{x}
$$

where :

$$
J_{q}=\left[\begin{array}{ccc}
B_{1} C_{1} \cdot u_{1} & 0 & 0 \\
0 & B_{2} C_{2} \cdot u_{2} & 0 \\
0 & 0 & B_{3} C_{3} \cdot u_{3}
\end{array}\right]
$$

and

$$
J_{x}=\left[\begin{array}{lll}
\left(B_{1} C_{1}\right)_{x} & \left(B_{1} C_{1}\right)_{y} & \left(B_{1} C_{1}\right)_{z} \\
\left(B_{2} C_{2}\right)_{x} & \left(B_{2} C_{2}\right)_{y} & \left(B_{2} C_{2}\right)_{z} \\
\left(B_{3} C_{3}\right)_{x} & \left(B_{3} C_{3}\right)_{y} & \left(B_{3} C_{3}\right)_{z}
\end{array}\right]
$$

Away from singular positions, the Jacobean matrix is:

$$
J=J_{x}^{-1} J_{q}
$$

\subsection{Research of the best position for prismatic joints}

We first reduced the research to the five arrangement depicted in Figure 2 since they seem to be "reasonable" solutions for practical implementation. At this stage, we decided to select a candidate for further study by resorting to two criterion:

$\checkmark$ The manipulability; we compared the worst condition number of the Jacobean matrix over the workspace (every possible candidate being analyzed at "its best", that is to say that we first determined the best geometry in each case, thanks to a rough optimization procedure);

$\checkmark$ The footprint; indeed, European car industry requires that machines placed along a production transfer line are smaller than $1.6 \mathrm{~m}$ in width.

In cases (a), (c) and (e) we have not been able to find an arrangement small enough to fit inside the footprint limits. We found that cases (b) and (d) offer the smallest footprint, while guaranteeing a good manipulability; however, note that the best manipulability is offered by case (e). Since the "footprint criterion" is absolutely mandatory, we discarded cases (a), (c) and (e). Finally ${ }^{1}$ we selected case (b) because it offers a possibly very large range of motion along $\mathrm{z}$ axis (depending only on the linear drives range of motion): this feature could be used to ease tool changeover.

\footnotetext{
1 This choice is not an ultimate answer by any means. This is a convenient answer in our specific case. The selection of a convenient arrangement depends a lot on the footprint requirements.
} 


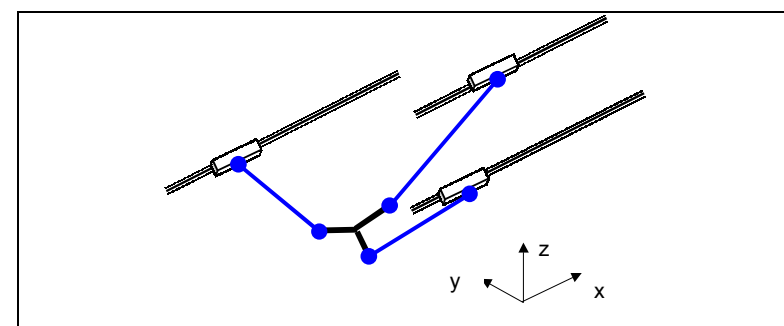

(a) Actuators parallel to $\mathrm{x}$ axis

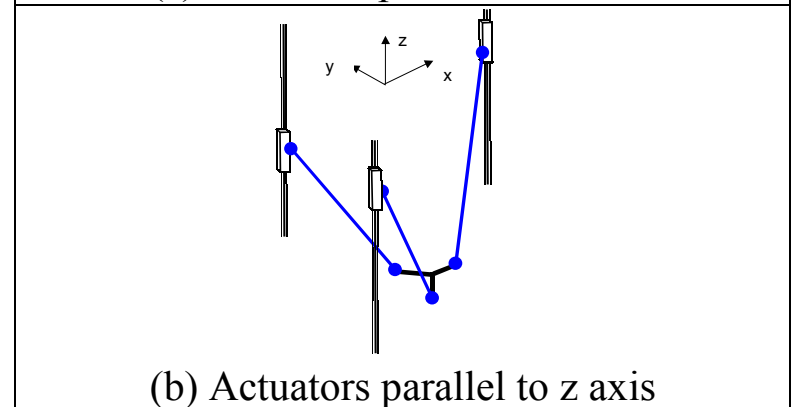

(b) Actuators parallel to $\mathrm{z}$ axis

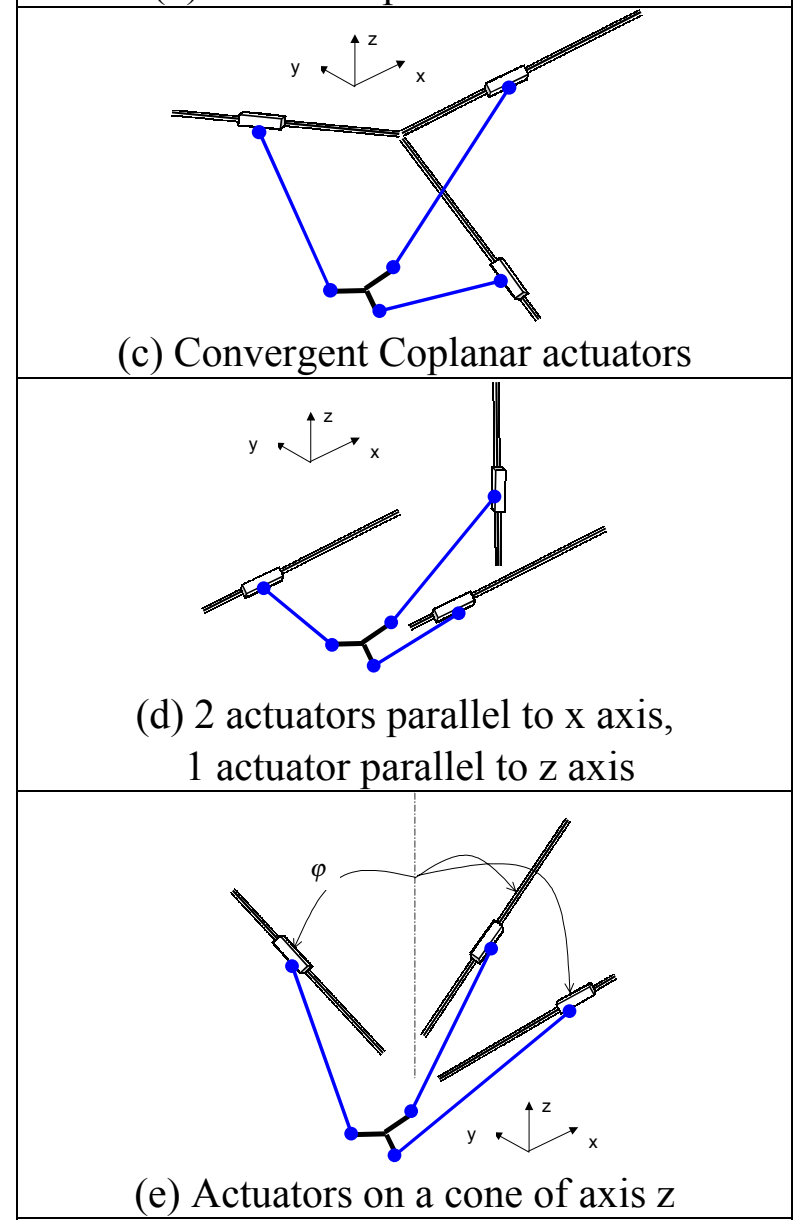

Figure 2 : Different possibilities for the location of the actuated joints.

\section{Detailed modeling of the selected arrangement}

Figure 3 shows a drawing of the chosen arrangement and Figure 4 shows the necessary parameters.

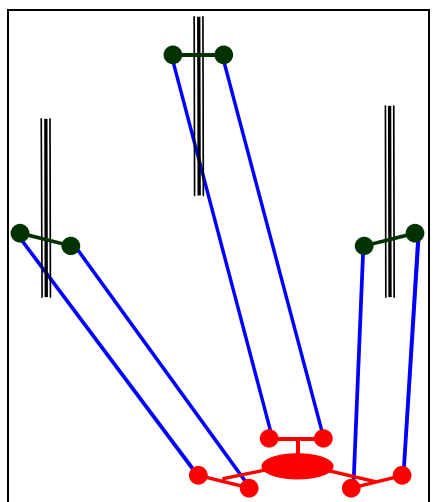

Figure 3 : Linear Delta scheme.

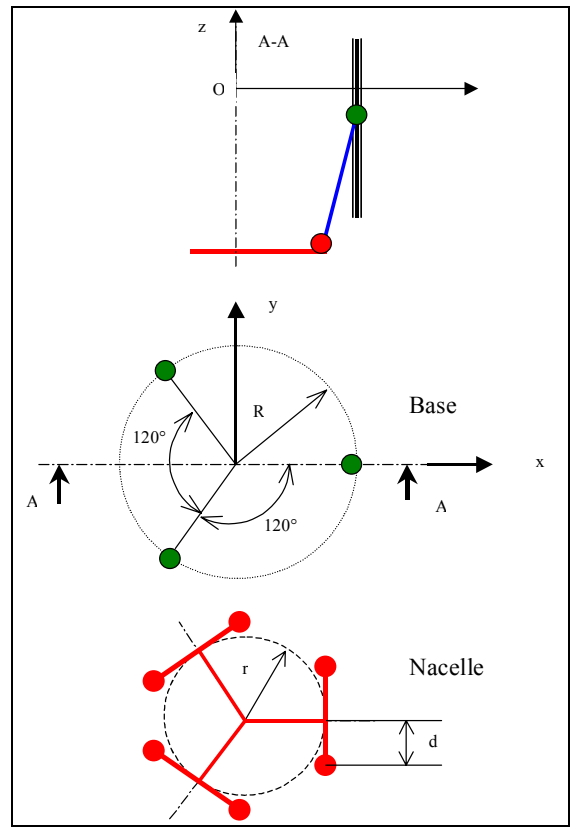

Figure 4 : Geometrical parameters.

\section{Inverse position relationship}

Thanks to parameters defined in Figure 4, the machine geometry is defined by: 


$$
\begin{aligned}
& {\left[\begin{array}{lll}
\boldsymbol{P}_{1} & \boldsymbol{P}_{2} & \boldsymbol{P}_{3}
\end{array}\right]=\left[\begin{array}{ccc}
R & -\frac{R}{2} & -\frac{R}{2} \\
0 & R \frac{\sqrt{3}}{2} & -R \frac{\sqrt{3}}{2} \\
0 & 0 & 0
\end{array}\right]} \\
& {\left[\begin{array}{lll}
C_{1} & C_{2} & C_{3}
\end{array}\right]_{R_{n}}=\left[\begin{array}{ccc}
r & -\frac{r}{2} & -\frac{r}{2} \\
0 & r \frac{\sqrt{3}}{2} & -r \frac{\sqrt{3}}{2} \\
0 & 0 & 0
\end{array}\right]} \\
& {\left[\begin{array}{lll}
\boldsymbol{u}_{1} & \boldsymbol{u}_{2} & \boldsymbol{u}_{3}
\end{array}\right]=\left[\begin{array}{lll}
0 & 0 & 0 \\
0 & 0 & 0 \\
1 & 1 & 1
\end{array}\right]}
\end{aligned}
$$

The three position equations become:

$$
\left\{\begin{array}{c}
(r-R+x)^{2}+y^{2}+\left(z-q_{1}\right)^{2}=L^{2} \\
\left((R-r) \frac{1}{2}+x\right)^{2}+\left((r-R) \frac{\sqrt{3}}{2}+y\right)^{2}+\left(z-q_{2}\right)^{2}=L^{2} \\
\left((R-r) \frac{1}{2}+x\right)^{2}+\left((R-r) \frac{\sqrt{3}}{2}+y\right)^{2}+\left(z-q_{3}\right)^{2}=L^{2}
\end{array}\right.
$$

The solution for inverse kinematics is:

$$
\left\{\begin{array}{l}
q_{1}=z+\sqrt{L^{2}-(r-R+x)^{2}-y^{2}} \\
q_{2}=z+\sqrt{L^{2}-\left((R-r) \frac{1}{2}+x\right)^{2}-\left((r-R) \frac{\sqrt{3}}{2}+y\right)^{2}} \\
q_{3}=z+\sqrt{L^{2}-\left((R-r) \frac{1}{2}+x\right)^{2}-\left((R-r) \frac{\sqrt{3}}{2}+y\right)^{2}}
\end{array}\right.
$$

\section{Forward position relationship}

The forward kinematics can be solved easily by deriving the roots of the following system:

$$
\left\{\begin{array}{c}
F z^{2}+2 G z+H=0 \\
y=A z+B \\
x=C z+D
\end{array}\right.
$$

where

$$
\begin{array}{cc}
A=\frac{\left(q_{2}-q_{3}\right)}{\sqrt{3}(r-R)} & B=\frac{q_{3}{ }^{2}-q_{2}{ }^{2}}{2 \sqrt{3}(r-R)} \\
C=\frac{2\left(q_{2}-q_{1}\right)-A(r-R) \sqrt{3}}{3(R-r)} & D=\frac{q_{1}^{2}-q_{2}{ }^{2}-B \sqrt{3}(r-R)}{3(R-r)} \\
E=r-R+B & F=A^{2}+C^{2}+1 \\
G=A E+C D-q_{1} & H=E^{2}+D^{2}+q_{1}{ }^{2}-L^{2}
\end{array}
$$

\section{Kinematics}

The two matrices involved in kinematics modeling can be written as follows :

$$
\begin{gathered}
\boldsymbol{J}_{\boldsymbol{x}}=\left[\begin{array}{ccc}
r-R+x & y & z-q_{1} \\
\frac{1}{2}(R-r)+x & \frac{\sqrt{3}}{2}(r-R)+y & z-q_{2} \\
\frac{1}{2}(R-r)+x & \frac{\sqrt{3}}{2}(R-r)+y & z-q_{3}
\end{array}\right] \\
\boldsymbol{J}_{\boldsymbol{q}}=\left[\begin{array}{ccc}
z-q_{1} & 0 & 0 \\
0 & z-q_{2} & 0 \\
0 & 0 & z-q_{3}
\end{array}\right]
\end{gathered}
$$

\section{Statics}

Let us note $\boldsymbol{f}_{\boldsymbol{m}}={ }^{t}\left[\begin{array}{lll}f_{m 1} & f_{m 2} & f_{m 3}\end{array}\right]$ the set of actuators forces, and $\boldsymbol{f}_{e}={ }^{t}\left[\begin{array}{lll}f_{x} & f_{y} & f_{z}\end{array}\right]$ the vector of external force acting on the traveling plate.

Thanks to the principle of virtual work, the static relationship between $\boldsymbol{f}_{\boldsymbol{m}}$ and $\boldsymbol{f}_{e}$ can be derived easily from the kinematics relationship, and be expressed as:

$$
\boldsymbol{f}_{m}={ }^{\boldsymbol{t}} \boldsymbol{J} \boldsymbol{f}_{\boldsymbol{e}}
$$

\section{Acceleration relationship}

Establishing the derivative of kinematics with respect to time leads to:

$$
\dot{J}_{x} \dot{x}+J_{x} \ddot{x}=\dot{J}_{q} \dot{q}+J_{q} \ddot{q}
$$




$$
\ddot{q}=J^{-1} \ddot{x}+J_{q}^{-1}\left(\dot{J}_{x}-\dot{J}_{q} J^{-1}\right) \dot{x}
$$

This relation introduces two new matrices:

$$
\dot{J}_{\boldsymbol{x}}=\left[\begin{array}{ccc}
\dot{x} & \dot{y} & \dot{z} \\
\dot{x} & \dot{y} & \dot{z} \\
\dot{x} & \dot{y} & \dot{z}
\end{array}\right] \text { and } \dot{\boldsymbol{J}}_{\boldsymbol{q}}=\left[\begin{array}{ccc}
\dot{z}-\dot{q}_{1} & 0 & 0 \\
0 & \dot{z}-\dot{q}_{2} & 0 \\
0 & 0 & \dot{z}-\dot{q}_{3}
\end{array}\right]
$$

Moreover, we can see that the actuators acceleration is the sum of two terms :

- $J^{-1} \ddot{\boldsymbol{x}}$, the acceleration of actuators due to the travelling plate acceleration.

- $\left(\dot{J}_{x}-\dot{J}_{q} J^{-1}\right) \dot{x}$, the acceleration of the actuators due to the constant speed of the travelling plate.

\section{Dynamics}

The rods are mainly stressed in tension and compression and can consequently be designed to have a low inertia, that can be neglected. Moreover, we assume that each rod mass, $m_{r}$, can be considered as being located at both rod ends.

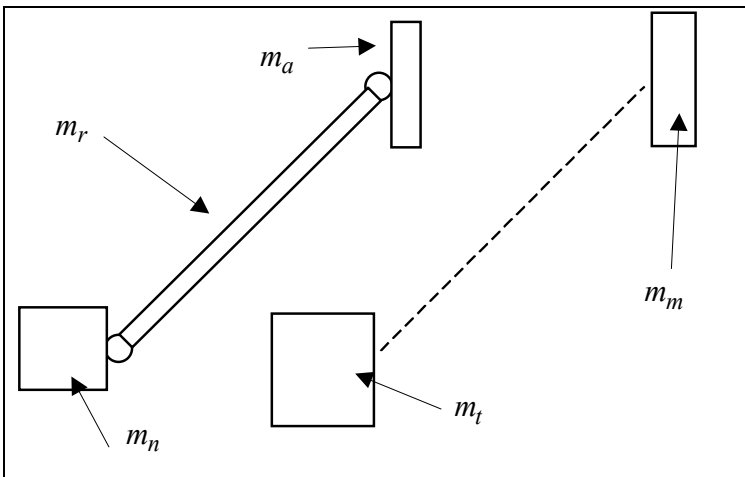

Figure 5: Assumption for a simpler dynamic model.

Thus we define the equivalent mass of the travelling plate, $m_{t}$, as the nacelle mass, $m_{n}$, plus half the mass of the six rods connected to it, and we define the equivalent mass of the motor moving part, $m_{m}$, as the mass of the actuator itself, $m_{a}$, plus half the mass of the two rods connected to it:

$$
m_{t}=m_{n}+6 \frac{m_{r}}{2}=m_{n}+3 m_{r}
$$

$$
m_{m}=m_{a}+2 \frac{m_{r}}{2}=m_{m}+m_{r}
$$

To move the travelling plate at pose $x$, at the speed $\dot{\boldsymbol{x}}$, with an acceleration $\ddot{\boldsymbol{x}}$ and external efforts $f_{e}$ acting on the travelling plate are, the actuator must compensate for:

- External forces applied on the travelling plate : ${ }^{\boldsymbol{}} \boldsymbol{J} \boldsymbol{f}_{\boldsymbol{e}}$

- Inertial effect of actuators moving parts: $m_{m} \ddot{\boldsymbol{q}}$

- Inertial effect of the travelling plate: $m_{t}{ }^{t} \boldsymbol{J} \ddot{\boldsymbol{x}}$

- sliding friction forces (not taken into account here).

The corresponding actuator force is then:

$$
\begin{aligned}
& \boldsymbol{f}_{\boldsymbol{m}}=\left(m_{t}{ }^{\boldsymbol{t}} \boldsymbol{J}+m_{m} \boldsymbol{J}^{-1}\right) \ddot{\boldsymbol{x}} \\
& +m_{m} \boldsymbol{J}_{\boldsymbol{q}}{ }^{-1}\left(\dot{\boldsymbol{J}}_{\boldsymbol{x}}-\dot{\boldsymbol{J}}_{\boldsymbol{q}} \boldsymbol{J}^{-1}\right) \dot{\boldsymbol{x}}+{ }^{\boldsymbol{t}} \boldsymbol{J} \boldsymbol{f}_{\boldsymbol{e}}
\end{aligned}
$$

\section{Forces in rods}

If we suppose that there is no friction in ball joints and if the mass of the bars is neglected, we can derive that the direction of the force in a bar is the same than the bar axis :

$$
\boldsymbol{f}_{i j}=f_{i j} \frac{\boldsymbol{B}_{\boldsymbol{i}} \boldsymbol{C}_{\boldsymbol{i}}}{L} \quad i=1, \cdots, 3 \quad j=1,2
$$

If the force on the travelling plate is $f_{e}$ and the torque is $\boldsymbol{m}_{\boldsymbol{e}}$, the travelling plate equilibrium can be written as follows :

$$
\left\{\begin{array}{c}
\sum_{i=1}^{3}\left(\sum_{j=1}^{2} \boldsymbol{f}_{\boldsymbol{i j}}\right)=\boldsymbol{f}_{\boldsymbol{e}} \\
\sum_{i=1}^{3}\left(\sum_{j=1}^{2} \boldsymbol{f}_{\boldsymbol{i j}} \times \boldsymbol{C}_{\boldsymbol{i j}} \boldsymbol{D}\right)=\boldsymbol{m}_{\boldsymbol{e}}
\end{array}\right.
$$




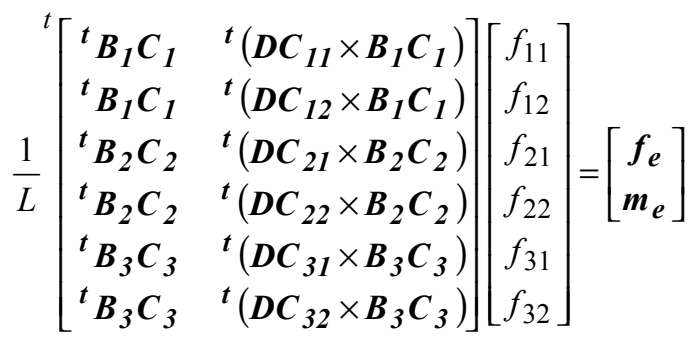

That is to say:

$$
J_{b} f=\left[\begin{array}{l}
f_{e} \\
m_{e}
\end{array}\right]
$$

So forces in bars are given by (if $\boldsymbol{J}_{\boldsymbol{b}}$ is not singular):

$$
f=J_{b}{ }^{-1}\left[\begin{array}{c}
f_{e} \\
m_{e}
\end{array}\right]
$$

\section{Machine stiffness.}

Stiffness is probably one of the most important feature for a machine-tool; evaluating a machine stiffness is always complex, in a practical point of view as well as at design stage where only difficult and time consuming FEA analysis can pretend to give reliable results.

However, it is important to give the designer a tool to compare solutions quickly. In the robotics community, people often evaluate roughly the quality of a design in terms of stiffness by resorting only to the analysis of actuators stiffness effects. We think that this could be really too simplified for our case, and we propose to take into account both the effect of actuators stiffness and rods stiffness.

We compute the small displacement of the travelling plate with external forces acting on it.

Actuator displacement influence.

Let us note $k_{m, i}$ the stiffness of $i^{\text {th }}$ motor, and $\Delta_{m, i}$ the small displacement of the motor when a force of modulus $f_{m, i}$ is acting on it; that is to say:

$$
f_{m, i}=k_{m, i} \Delta_{m, i}
$$

In a matrix format, this leads to:

$$
\Delta_{m}=K_{m}^{-1}{ }^{t} J f_{e}
$$

where:

$$
\begin{gathered}
\boldsymbol{f}_{\boldsymbol{m}}={ }^{t}\left[\begin{array}{lll}
f_{m, 1} & f_{m, 2} & f_{m, 2}
\end{array}\right] \\
\boldsymbol{\Delta}_{\boldsymbol{m}}={ }^{t}\left[\begin{array}{lll}
\Delta_{m, 1} & \Delta_{m, 2} & \Delta_{m, 2}
\end{array}\right] \\
\boldsymbol{K}_{\boldsymbol{m}}=\operatorname{diag}\left(\left[\begin{array}{lll}
k_{m, 1} & k_{m, 2} & k_{m, 2}
\end{array}\right]\right)
\end{gathered}
$$

and the resulting small displacement of the travelling plate due to motors displacement is:

$$
\Delta x_{m}=J K_{m}^{-1}{ }^{t} J f_{e}
$$

Rods deformation influence.

In the same way, a relationship exists between the force acting on $i^{\text {th }}$ rod, $f_{r, i}$, its stiffness $k_{r, i}$ and a change, $\Delta_{r, i}$, of its nominal length:

$$
f_{r, i}=k_{r, i} \Delta_{r, i}
$$

Assuming that Hooke's law is acceptable for the rods, we have:

$$
k_{r, i}=\frac{S_{i} E_{i}}{L_{i}}
$$

where:

$E_{i}$ is Young's modulus of the rod,

$S_{i}$ is the surface of the rod cross section, $L_{i}$ is the rod nominal length.

Again in a matrix format, this leads to:

$$
\Delta_{r}=\boldsymbol{K}_{r}^{-1} \boldsymbol{f}_{\boldsymbol{r}}
$$

where:

$$
\begin{gathered}
\boldsymbol{f}_{\boldsymbol{r}}={ }^{t}\left[\begin{array}{lll}
f_{r, 1} & \cdots & f_{r, 6}
\end{array}\right] \\
\boldsymbol{\Delta}_{\boldsymbol{r}}={ }^{t}\left[\begin{array}{lll}
\Delta_{r, 1} & \cdots & \Delta_{r, 6}
\end{array}\right] \\
\boldsymbol{K}_{\boldsymbol{r}}=\operatorname{diag}\left(\left[\begin{array}{lll}
k_{r, 1} & \cdots & k_{r, 6}
\end{array}\right]\right)
\end{gathered}
$$


We know that:

$$
f_{r}=J_{b}^{-1}\left[\begin{array}{c}
f_{e} \\
m_{e}
\end{array}\right]
$$

thus the resulting small displacement of the travelling plate due to rods deformation is::

$$
\left[\begin{array}{l}
\Delta x_{r} \\
\Delta \alpha_{r}
\end{array}\right]=K_{r}^{-1} t^{t}\left(J_{b}^{-1}\right) J_{b}{ }^{-1}\left[\begin{array}{c}
f_{e} \\
m_{e}
\end{array}\right]
$$

Where $\Delta x_{r}$ is the small displacement and $\Delta \alpha_{r}$ is the angular small displacement of the travelling plate.

Total displacement.

Assuming that all displacements are small, they can be added as follows to get the displacement of a point $E$ :

$$
\Delta x=\Delta x_{m}+\Delta x_{r}+E D \otimes \Delta \alpha_{r}
$$

\section{Results}

In this section some results obtained using the previous relationships are shown, assuming that all rods have the same length $L$ and for the selected arrangement.

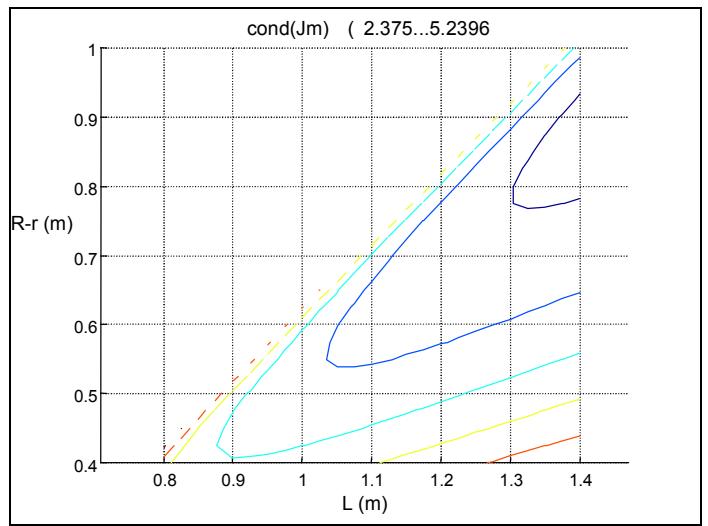

Figure 6:Level curves for the worst condition number versus $R-r$ and $L$.

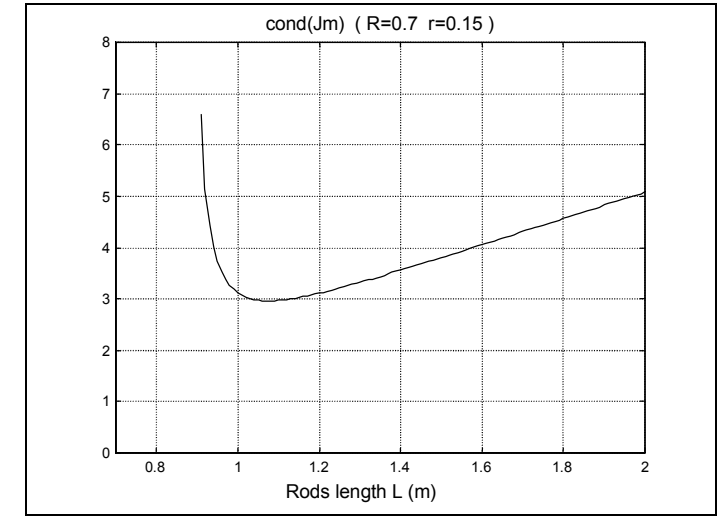

Figure 7:Evolution of condition number for a given $R-r$ versus $L$.

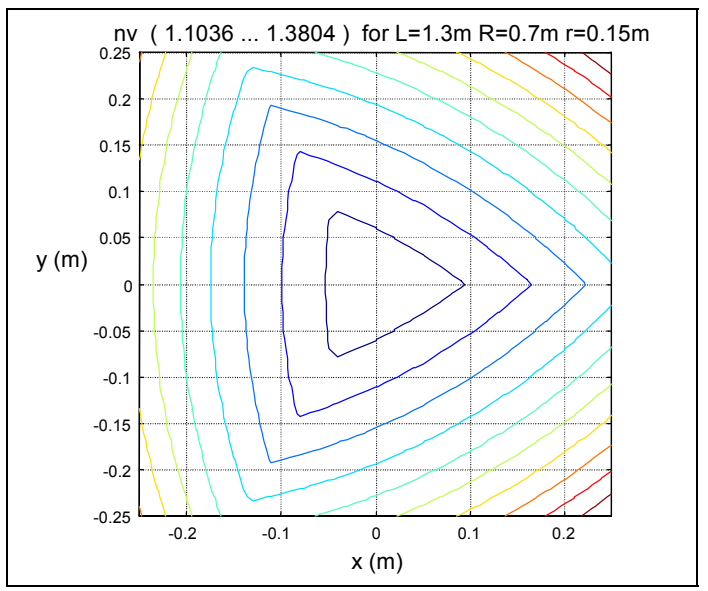

Figure 8:Level curves for the velocity transformation in $(x, y)$ plane.

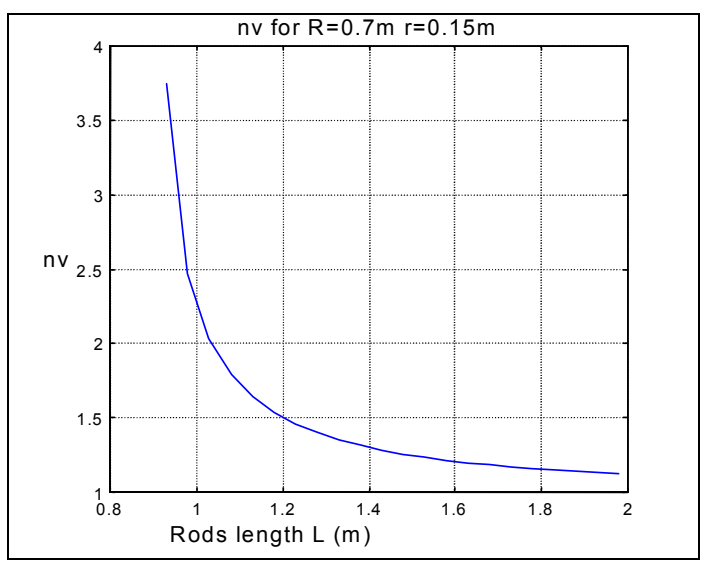

Figure 9:Evolution of velocity transformation versus $L$ for a given $R-r$. 


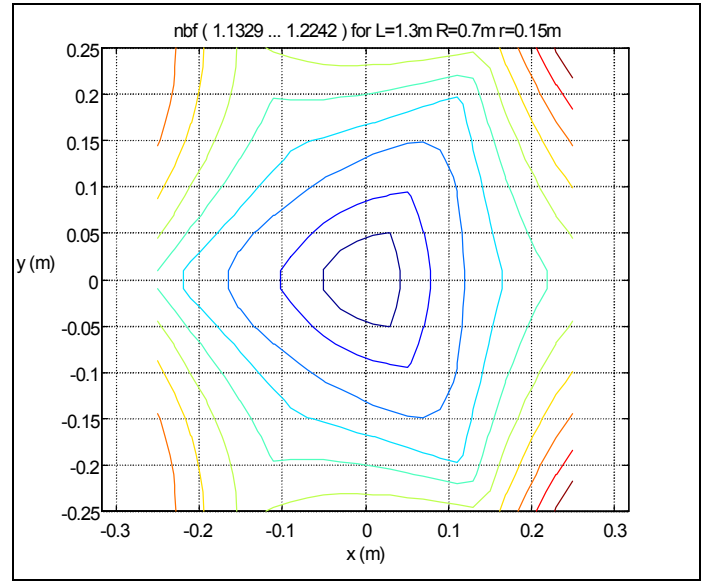

Figure 10:Level curves for force transformation in $(x, y)$ plane for a pure force $f_{e}$.

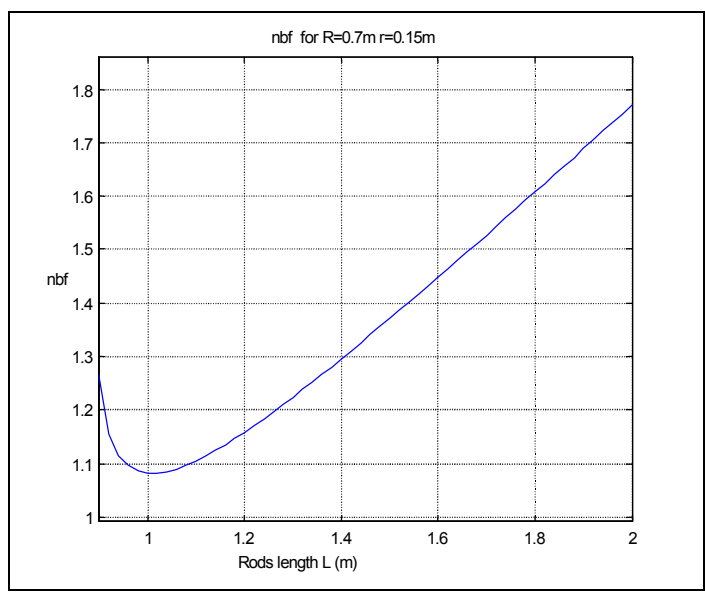

Figure 11:Evolution of force transformation for a pure force $f_{e}$ versus $L$ for given $R, r$ and $d$.

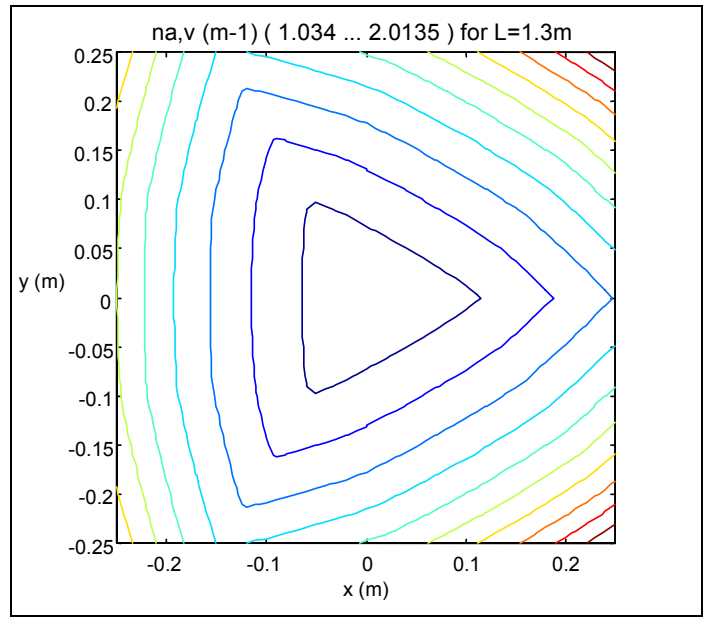

Figure 12:Level curves for nacelle velocity to actuators acceleration transformation in $(x, y)$ plane.

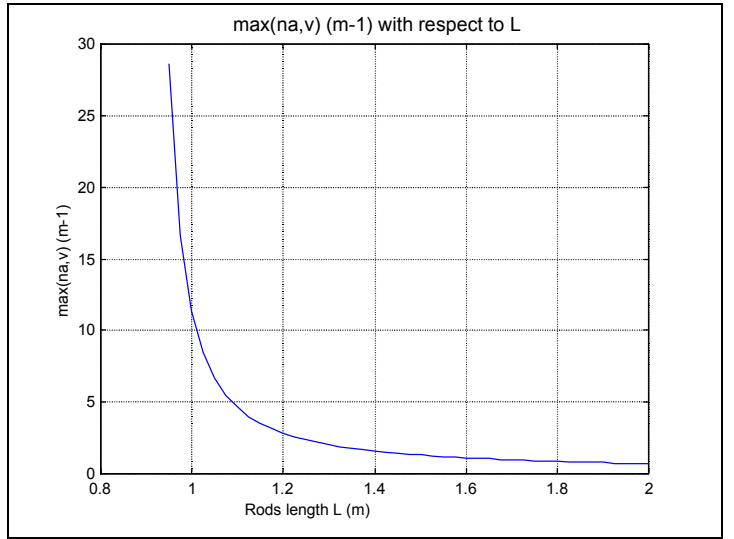

Figure 13:Evolution of nacelle velocity to actuators acceleration transformation versus $L$ for a given $R-r$.

These results show that, for a given $R-r$, there is an optimal value for $L$ depending on the chosen criterion (worst condition number, velocity transformation, force transformation in rods...).

The rotational symmetry of the design is found in the level curves in $(x, y)$ plane (figures 8, 10 and 12). The machine behavior does not depend on the value of $z$ if all the points of the corresponding $(x, y)$ plane are reachable.

In one hand, figures 9 and 13 show that, for velocity transformation considerations, $L$ must be taken at the maximum value. On the other hand, for force transformation (figure 11), the chosen $L$ must be small. These results are an illustration of the duality between force and velocity. So the choice of the machine dimensions is a balance with respect to the desired characteristics.

\section{Conclusion}

We have chosen a parallel structure for high speed point-to-point displacement and simple machining For this architecture we developed detailed models. For a given drilling task case one can use these models to find the optimal parameters for the machine geometry. Then one can compute the features of some elements of the machine (actuators thrust, diameter of rods). 
This has been successfully applied to a machine-tool prototype developed by RenaultAutomation Comau, a machine-tool company in Castres, France: Urane $\mathrm{Sx}$ is capable of acceleration between 3.5 and $5.0 \mathrm{~g}$, with a velocity of $120 \mathrm{~m} / \mathrm{min}$ while being equipped with a fully functional high-speed spindle (Figure 14)

\section{References}

[1] Machine Automation, No 21, May 1999

[2] Clavel R. DELTA, a fast robot with parallel geometry. In 18th International Symposium on Industrial Robot, pages 91-100, Lausanne, 2628 April 1988.

[3] Tsai L-W. Kinematics of a three-dof platform with three extensible limbs. In J.
Lenarcic V. Parenti-Castelli, editor, Recent Advances in Robot Kinematics, pages 401-410. Kluwer, 1996.

[4] Hervé J.M. Group mathematics and parallel link mechanisms. In IMACS/SICE Int. Symp. on Robotics, Mechatronics, and Manufacturing Systems, pages 459-464, Kobe, 16-20 September 1992.

[5] Hervé J.M. Group mathematics and parallel link mechanisms. In 9th World Congress on the Theory of Machines and Mechanisms, pages 2079-2082, Milan, 30 August, 1995.

[6] Mitova T. and Vatkitchev A. Analysis of a closed space mechanism with three degree of mobility. In XI COBEM, Rio de Janeiro, 1991.

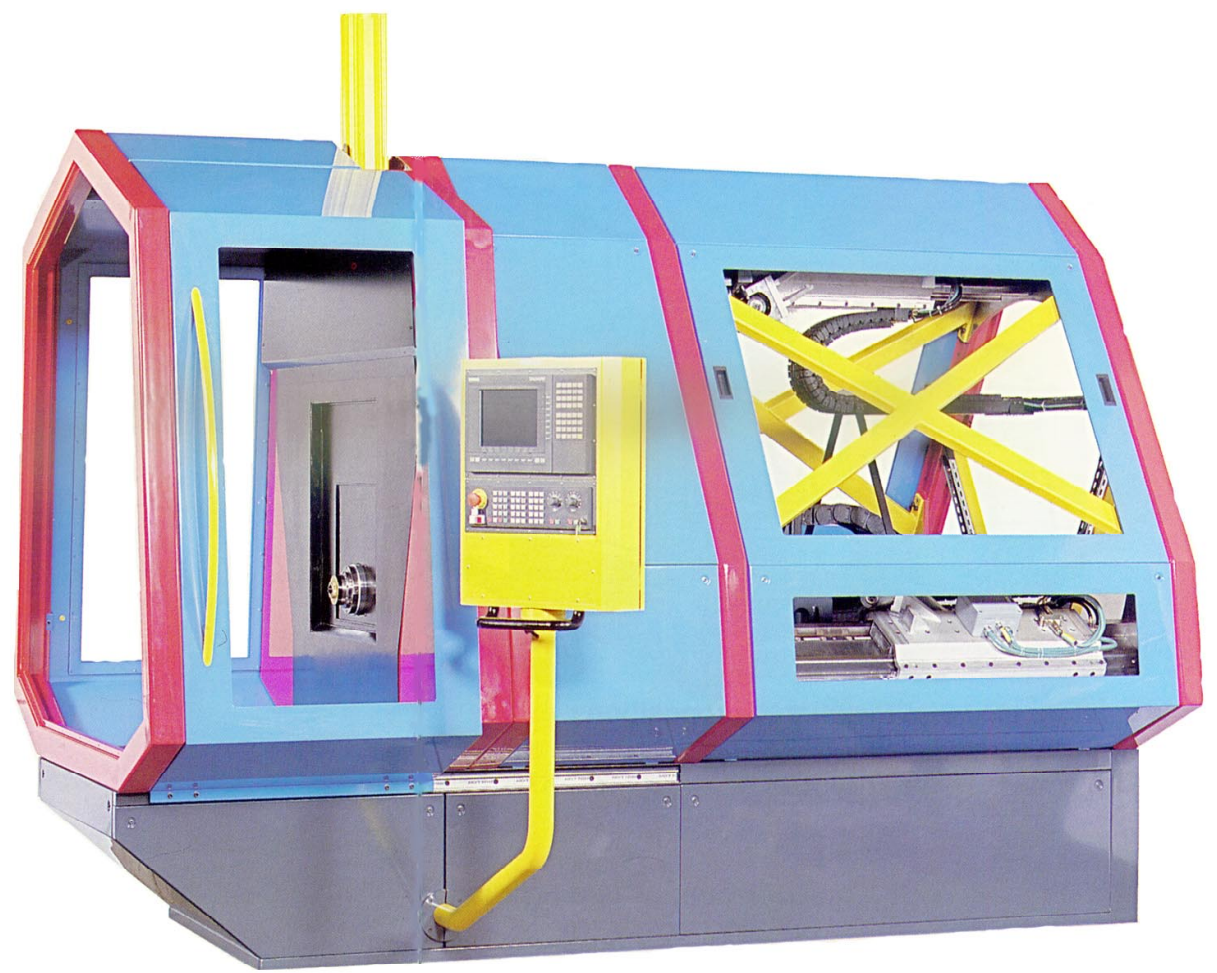

Figure 14. Urane Sx first prototype. 